\title{
Monthly Maximum load Demand Forecasting for Sulaimani Governorate Using Different Weather Conditions Based on Artificial Neural Network Model
}

\author{
Najat Hassan Abdulkareem \\ MoEl (Ministry of Electricity-KRG), Electricity control center, Sulaimani/Iraq
}

\section{A B S T R A C T}

Medium-term forecasting is an important category of electric load forecasting that covers a time span of up to 1 year ahead. It suits outage and maintenance planning, as well as load switching operation. There is an on-going attention toward putting new approaches to the task. Recently, artificial neural network has played a successful role in various applications. This paper is presents a monthly peak load demand forecasting for Sulaimani (located in North Iraq) using the most widely used traditional method based on an artificial natural network, the performance of the model is tested on the actual historical monthly demand of the governorate for the years 2014-2018. The standard mean absolute percentage error (MAPE) method is used to evaluate the accuracy of forecasting models, the results obtained show a very good estimation of the load. The MAPE is 0.056 .

Index Terms: Actual Load, Artificial Neural Network, Midterm Monthly Load Forecast, Multilayer Perceptron, Predicted Load. Yearly Ahead

\section{INTRODUCTION}

Power system planning starts with the forecast of load requirements. With the fast growth of power systems networks and increase in their complexity, many factors have become influential in electric power generation, demand, or load management. The forecasting of electricity demand has been one of the major research fields in electrical engineering. Massive investment decisions for network reinforcement and expansions are made based on the load forecast. Hence, it is necessary to have accurate load forecast to carry out proper

\begin{tabular}{|l|l|}
\hline \multicolumn{2}{|c|}{ Access this article online } \\
\hline DOI: 10.21928/uhdjst.v4n2y2020.pp10-17 & $\begin{array}{l}\text { E-ISSN: 2521-4217 } \\
\text { P-ISSN: 2521-4209 }\end{array}$ \\
\hline $\begin{array}{l}\text { Copyright @ 2020 Abdulkareem. This is an open access article } \\
\text { distributed under the Creative Commons Attribution Non-Commercial } \\
\text { No Derivatives License 4.0 (CC BY-NC-ND 4.0) }\end{array}$ \\
\hline
\end{tabular}

planning [1], [2]. Although, load forecasting is one of the major factors for economic operation of power systems. Future load forecasting is also important for network planning, infrastructure development, and so on.

Power system load forecasting can be classified into three categories, namely, short-term, medium-term, and long-term load forecasting. The periods for these categories are not defined clearly in literature [2].

Thus, different authors use different time periods to define these categories. However, roughly, short-term load forecasting (STLF) covers hourly to weekly forecast. These forecasts are often needed for day-to-day economic operation of power generating units [3].

Midterm load forecasting has period time in 3 months-3 years, maintenance of plants and networks is often roofed in these types of forecast.

Corresponding author's e-mail: Najat Hassan Abdulkareem, MoEl (Ministry of Electricity-KRG), Electricity control center, Sulaimani//raq. E-mail: Qaradakhi@gmail.com

Received: 03-05-2020

Accepted: 07-01-2020

Published: 07-05-2020 
Long-term forecasting, on the other hand, deals with forecast from few months to 1 year. It is primarily intended for capacity expansion plans, capital investments, and corporate budgeting. These types of forecasts are often complex in nature due to future uncertainties such as planning and extension of existing power system networks for both the utility and consumers required long-term forecasts [3].

The system load is a random non-stationary process composed of thousands of individual components. The system load behavior is influenced by a number of factors, which can be classified as economic factors, time, weather, and random effects. The economic environment in which the utility operates has a clear effect on the electric demand consumption patterns, such as the service area demographics, level of industrial activity, and changes in farming sector [4].

For the present, there are many algorithms for load forecasting in the computation intelligence such as fuzzy logic (Fs) [5], neural network, and genetic [6]. Many research purposed the article for load forecasting in the power system field: STLF using autoregressive integrated moving average (ARIMA) and artificial neural network (ANN) method based on non-linear load, a novel method approach to load forecasting using regressive model and $\mathrm{ANN}$ the combination of $\mathrm{ANN}$, genetic algorithm, and Fs method is proposed for adjusting STLF of electric system. Genetic algorithm is used for selecting better rules and backpropagation algorithm is also for this network, papers show that more accuracy results and faster processor than other forecasting methods [7].

The aim of this paper is to provide a monthly peak demand forecast for Sulaimani Governorate (located in North Iraq). This forecast is of special importance to this region because of the present shortage of generating capacity and the need for extensive load shedding. It is thus important to estimate what the near-term demand will be, especially in the peak demand months, as a key input in determining the availability of enough generating capacity to meet the demand [8].

Sulaimani is one of the four Northern Governorates of Iraq (Iraqi Kurdistan Region). It is bounded by Iran; Erbil Governorate and Kirkuk Governorate. The land area of the governorate is about $18,240 \mathrm{Sq} . \mathrm{Km}$. The city of Sulaimani is located in $335 \mathrm{~km}$ northeast to Baghdad.

This study is tried to find out the way to forecast the electrical power load in Sulaimani Governorate power distribution network using ANN with the help of MATLAB software. The input is involved three feature; precipitation, temperature, and humidity. These three parameters effect on power consumption directly. Mean square error is used as performance measure.

The method of learning in ANN which is used in this paper is feed forward backpropagation. Finally, by comparing different cases is tried to find best solution to estimate the load demand.

This dataset consists of 5 years of average monthly load collected from the Electricity Control Center (ECC) of Kurdistan Region in Iraq. The data are accumulated daily and involve the maximum electrical demand load in Mega Watt (MW) from January 1, 2014, to October 31, 2018. The dataset is also accompanied by temperature, humidity, and precipitation collected, which can be used to forecast the demand.

\section{THE LOAD PROFILE OF SULAIMANI GOVERNORATE}

There is a severe shortage of electricity supply in Sulaimani Governorate. Main sources of electricity supply are Dokan hydropower station $(5 \times 80 \mathrm{MW})$, Derdandikhan hydropower station $(3 \times 83 \mathrm{MW})$, Sulaimani combined cycle gas power plant which is consists of 10 units ( 8 simple cycle $125 \mathrm{MW}$ per unit and 2 combined cycle $250 \mathrm{MW}$ per unit), Tasluja Heavy Fuel Power Plant (51 MW), and Bazyan Gas Power Plant (4×125 MW), which is also supply Erbil and Dhuk Governorates. (For explain this: All of this power stations cannot work in its full capacity. And the generate power also go to Erbil and Duhok, therefore, the Sulaimani Governorate demand every time is bigger than the power generation and that is the problem).

However, the available supply from the above sources dose not meets the power requirements in the governorate. Consumers are provided for a very short duration, sometimes $10 \mathrm{~h}$ per day depending on the generation capacity. Monthly energy consumption demand (unit in MWh) data is recorded form (ECC) Kurdistan Region from 2014 to 2018. Fig. 1 shows the relationship between energy consumption demand and time (corrected divide each year into 12 months).

We consider the period from 2014 to 2018 to establish the parameters in forecast model. The original signal (behavior) of energy consumption demand is shown in Fig. 1. It grew the higher demand every year. The maximum demand is occurred on months $11,12,1,2,6,7$, and 8 and minimum demand is occurred in months 3, 4, 5, 9, and 10. Kurdistan regions climate is characterized by cool winters and hot summers. This extreme temperature swing affects the demand and 
producing a typical summer and winter peak demand periods each year. The monthly peak temperature profile for the years 2014-2018 is shown in Fig. 2.

\subsection{Forecasting Methods}

In terms of lead time, load forecasting is divided into four categories:

- Long-term forecasting with the lead time of more than 1 year

- Midterm forecasting with the lead time of 1 week-1 year

- STLF with the lead time of 1 day-1 week

- Very STLF with the lead time shorter than 1 day.

The research approaches of load forecasting can be mainly divided into two categories:

Statistical methods and artificial intelligence methods. In statistical methods, equations can be obtained showing the relationship between load and its relative factors after training the historical data, while artificial intelligence methods try to imitate human beings way of thinking and reasoning to get knowledge from the past experience and forecast the future load. Some main STLF methods are introduced as follows. Regression methods regression is one of most widely used statistical techniques. For load forecasting, regression methods are usually employed to

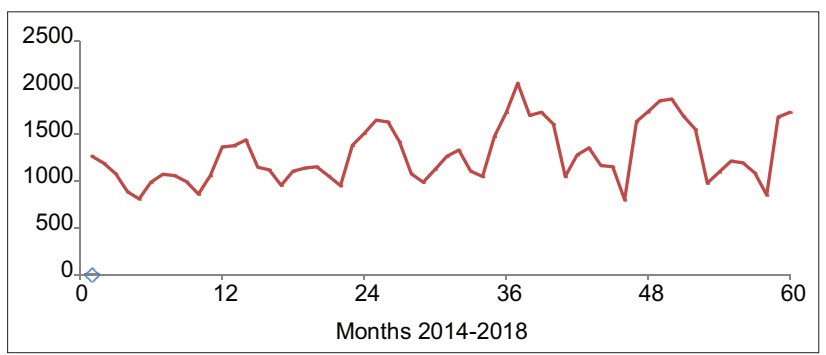

Fig. 1. Monthly energy consumption demand of Sulaimani city from January 2014 to December 2018 (DCC).

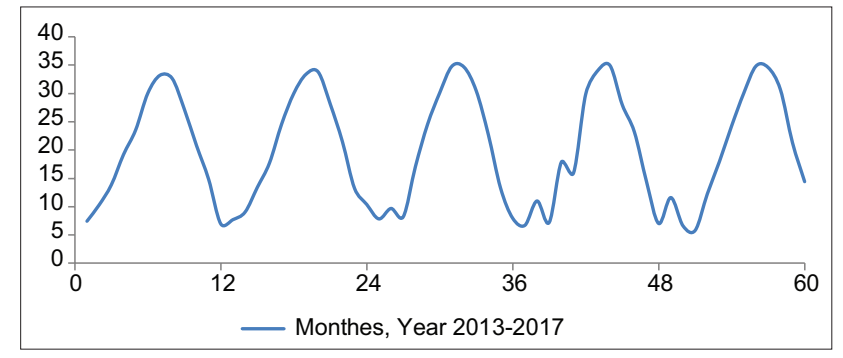

Fig. 2. Average temperature of Sulaimani Governorate from 2013 to 2017.

References:

1. http://www.motac.gov.krd/news.aspx?id=1226.

2. The (Sulaimani Directory of Meteorological and Seismology Data) Page on Facebook: shorturl.at/gmyY9. model the relationship of load consumption and other factors such as weather, day type, and customer class. Time series methods are based on the assumption that the data have an internal structure, such as autocorrelation, trend, or seasonal variation. The methods detect and explore such a structure. Time series have been used for decades in such fields as economics, digital signal processing, as well as electric load forecasting. In particular, ARMA (autoregressive moving average), ARIMA, and ARIMAX (ARIMA with exogenous variables) are the most often used classical time series methods. ARMA models are usually used for stationary processes while ARIMA is an extension of ARMA to non-stationary processes. ARMA and ARIMA use the time and load as the only input parameters. Since load generally depends on the weather and time of the day, ARIMAX is the most natural tool for load forecasting among the classical time series models. Similar day approach, this approach is based on searching historical data for days within 1,2 , or 3 years with similar characteristics to the forecast day. Similar characteristics include weather, day of the week, and the date. The load of a similar day is considered as a forecast. Instead of a single similar day load, the forecast can be a linear combination or regression procedure that can include several similar days. The trend coefficients can be used for similar days in the previous years. Expert systems are heuristic models, which are usually able to take both quantitative and qualitative factors into account. A typical approach is to try to imitate the reasoning of a human operator. The idea is then to reduce the analogical thinking behind the intuitive forecasting to formal steps of logic. A possible method for a human expert to create the forecast is to search in history database for a day that corresponds to the target day with regard to the day type, social factors, and weather factors. Then, the load values of this similar day are taken as the basis for the forecast.

Fs is a generalization of the usual Boolean logic used for digital circuit design. An input under Boolean logic takes on a value of "True" or "False." Under Fs an input is associated with certain qualitative ranges. For instance, the temperature of a day may be "low," "medium," or "high." Fs allows one to logically deduce outputs from fuzzy inputs. In this sense, Fs is one of a number of techniques for mapping inputs to outputs. Among the advantages of the use of Fs are the absence of a need for a mathematical model mapping inputs to outputs and the absence of a need for precise inputs. With such generic conditioning rules, properly designed Fs systems can be very robust when used for forecasting. Integration of different algorithms are many presented methods for (STLF), it is natural to combine the results of several methods. One simple way is to get the average value of them, which can lower the risk of individual unsatisfactory prediction. A more complicated and reasonable 
way is to get the weight coefficient of every forecasting method by reviewing the historical prediction results. The comprehensive result is deduced by weighted average method.

\subsection{Forecasting Requirements}

This subsection lists and describes the requirements to develop a user friendly and a good load forecasting tool. A good load forecasting tool should fulfill the requirement of accuracy, fast speed, friendly interface, and automatic data access. Accuracy the most important requirement of designing a load forecasting tool is its prediction accuracy. As mentioned before, good accuracy is the basis of economic dispatch, system reliability, and electricity markets. The main goal of this paper is to make the forecasting result as accurate as possible. Fast speed employment of the latest historical data helps to increase the accuracy. When the deadline of the forecasted result is fixed, the longer the runtime of the forecasting program is, the earlier historical data can be employed by the program. Therefore, the speed of the forecasting is a basic requirement of the forecasting program. Programs with too long training time should be abandoned and new techniques shortening the training time should be employed.

Friendly interface the graphical user interface of the load forecasting tool should be easy, convenient, and practical. The users can easily define what they want to forecast, whether through graphics or tables. The output should also be with the graphical and numerical format, in order that the users can access it easily.

Automatic data access the historical data is stored in the database. The load forecasting tool should be able to access it automatically and get the needed data.

\subsection{Advantages and Disadvantages of Load Forecasting \\ 2.3.1. Advantages}

1. It enables the utility company to plan well since they have an understanding of the future consumption or load demand

2. Useful to determine the required resources such as fuels required to operate the generating plants as well as other resources that are required to ensure uninterrupted and yet economical generation and distribution of the power to the consumers. This is important for all short-, medium-, and long-term planning

3. Planning the future in terms of the size, location, and type of the future generating plant is the factors which are determined by the help of load forecasting

4. Provides maximum utilization of power generating plants. The forecasting avoids under generation or over generation

\subsubsection{Disadvantages}

1. It is not possible to forecast the future with accuracy. The qualitative nature of forecasting, a business can come up with different scenarios depending on the interpretation of the data

2. Organizations should never rely $100 \%$ on any forecasting method

However, an organization can effectively use forecasting with other tools of analysis to give the organization the best possible information about the future.

3. Making a decision based on a bad forecast can result in financial ruin for the organization, so the decisions of an organization should never base solely on a forecast

\section{LOAD FORECASTING USING ANN}

A large variety of artificial intelligence and statistical techniques has been developed for load forecasting. Some of the methods such as similar day approach, regression methods, time series, neural network, expert systems, and Fs are used nowadays, among them, ANN is one of a good choice to apply for the load demand forecasting problem because this technique is not requiring explicit models to represent the complex relationship between the load demand and factors. ANN methods are particularly attractive, as they have the ability to handle the non-linear relationships between load and the factors affecting it directly from historical data. ANNs use a dense interconnection of computing nodes to approximate non-linear functions each node constitutes a neuron and performs the multiplication of the input signals by constant weight, sums up the results, and maps the sum to a nonlinear activation function, the result is then transferred to its input [9].

\subsection{Basic Theory - Feed Forward Backpropagation}

In this paper, we use backpropagation feed forward neural network to model the problem. Fig. 1 shows an example of ANN structure; each neural network has at least three layers, input layer, a hidden layer, and output layer. In a typical multilayer network, the input units which are denoted by Xi are connected to all hidden layer units which are defined by $\mathrm{Yj}$ and the hidden layer units are connected to all output layer units which are denoted by Zk. The elements Wij or Vij of the weight matrix associate the weight of each connection between the input to hidden and hidden to output layer units. The hidden and output layer units also receive signals from weighted connections (bias) from units whose values are always 1.

In each output and hidden units, the incoming signals from the previous layer sum together and apply an activation function to form the response of the net for a given input pattern. 


$$
\begin{aligned}
& x_{i} \text { input } i=1,2, \ldots n \\
& (Y j)=f b j+\sum X i W i j \\
& (Z k)=f b k+\sum Y j V j k
\end{aligned}
$$

To determine the error, each output will be compare its output $(\mathrm{Zk})$ with the actual output value which is identified by $\mathrm{dk}$. Then, according to the calculated error, $\delta k$ will be determined. $\delta k$ is a factor which is used to distribute the error at Zk back to all units in the previous layer.

$$
\delta k=f^{\prime}(Z k)(d k-Z k)
$$

Zk: Calculated output for each layer

$D k:$ Actual output

$\delta k$ : the factor for calculation the errors.

Factor $\delta j$ will be computes for each hidden unit. This factor is a weighted sum of all the back propagated delta terms from units in the previous layer multiplied by the derivative of the activation function for that unit.

$$
\delta j=f^{\prime}(Y j) \sum \delta k V j k
$$

In the next step, the new value of bias and each element of weight matrix will be calculated where $\eta$ is a learning rate coefficient that is given a value between 0 and 1 at the start of training.

$$
\begin{aligned}
& b j(\text { new })=b j(\text { old })+\eta \delta j \\
& W i j \text { (new) }=W i j \text { (old })+\eta \delta j \mathrm{Xi}
\end{aligned}
$$

In each irritation, it will be checked if the stop condition is occurred or not. The stop condition can be reaching error threshold, defined irritation, etc. [10], [11].

\subsection{Problem Formulation and Methodology}

The following steps have been followed by the investigator to formulate the above said problem:

i. First of all historical weather and load data is scrutinized. All monthly and daily predictions have been read

ii. Then, database has been created by the investigator for developing load forecasting model

iii. Accordingly, temperature and humidity have been differentiated as average value

iv. Furthermore, the rainy season and predicted rainfall have been considered for making the algorithm for midterm load forecasting

v. After this classification some ANN technique has been used to train these input variables for getting the expected outcome. System has been simulated with the help of MATLAB/SIMULINK

vi. Then, percentage error (PE) has been calculated for the given forecasting model.

\subsection{Accuracy of Forecasted}

To evaluate forecasting accuracy of the whole procedure, the following indices have been calculated in Equations (8 and 9), mean square error (MSE) for each month of forecasting:

$$
M S E=\sum_{i=1}^{M} \frac{\left(\text { Actiual }_{i}-\text { Forecast }_{i}\right)^{2}}{M}
$$

Mean absolute percentage error (MAPE) given by:

$$
M A P E=\frac{1}{M} \sum_{i=1}^{M} \frac{\left(\text { Actiual }_{i}-\text { Forecast }_{i}\right)^{2}}{\text { Actiual }_{i}} \times 100
$$

Where, Actual is the real value of monthly load demand at the each year, Forecasted is the forecasted value in the same year, and $M$ is month [12], [13].

After forecasting the load patterns for each test month, these forecasts were compared with the real load data, and the average error percentages were calculated. In comparing different models, the average percentage forecasting error is used as a measure of the performance. The reason for using the average percentage error is the fact that its meaning can be easily understood. It is also the most often used error measure in the load forecasting literature used as reference of this work and therefore allows for some comparative considerations (although the results are not directly comparable in different situations).

However, when both measures calculated on some test models with relatively small errors, the orders of preference were in practice the same with both measures. Therefore, the average forecasting error will be used throughout this work. In case of the monthly forecast, the training algorithms of gradient backpropagation and Levenberg-Marquardt were compared, whereas in case of the monthly forecast, the simulation was done. Although Levenberg-Marquardt is a very fast training algorithm, it often has given fairly inaccurate results due to the large approximations it makes while calculating the Hessian matrix [14], [15].

From years 2014 to 2018 were chosen in different seasons.

of the year. The load of each month of the dataset was forecast without using the actual load data of that month. 
Thereby, the monthly forecasting model was applied for each test of monthly peak demand recursively for all months in the year. After forecasting the load patterns for each test month, these forecasts were compared with the real load data, and the average error percentages were calculated.

\section{CASE STUDY AND TEST RESULTS}

The present study develops midterm electric load forecasting using neural network; based on historical series of power demand, the neural network chosen for this network is feed forward network, case study and test results will be present in this section by following:

\subsection{Case Study}

This study used the historical information or data for proposing ANN model to load forecasting as following:

Monthly energy consumption demand $(M W h)$, the humidity $(H)$, precipitation $(\mathrm{P})$, and temperature $(\mathrm{T})$. All of data information are recorded from 2014 to 2018.

Table 1 shows the block model for 2 years ahead demand forecasting which have four inputs: The historical load demands from -12 months to -48 months, maximum temperature from 1 months to -48 months, humidity from 1 months to -84 months, and precipitation 1 months to -48 months. These are feature inputs to ANN. The output of this model is the load demand of Sulaimani Governorate that is +24 months ahead or 2 years ahead. Note that: The historical load Demands: year 2018 (0), 2017 (-12), 2016 (-24), 2015 (-36), and 2014 (-48).

Various network models based on multilayer backpropagation feed forward architecture are tested with different designs and different configurations of hyperparameters (one and two) hidden layer with neurons of 10,12,15, and different transfer functions. After several trials, the near-optimal values have been taken. The information about different cases (for 1 month January 2014, for example) is described in Table 1. A backpropagation network with momentary and with adaptive

\section{TABLE 1: The model for 2 years ahead forecasting}

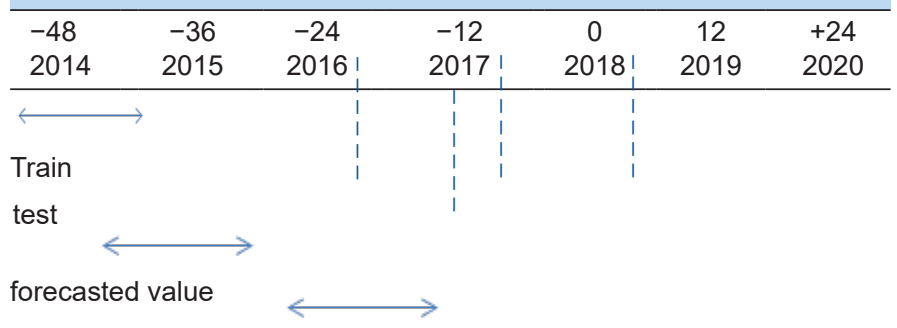

learning rate was trained and the neural network can forecast future load 1 day peak load per month ahead given in various inputs to the network. A sigmoid transfer function was used in the hidden layer while a linear transfer function was used in. It has been observed that using 12 neurons and transfer function of logsig gave less percentage error for nearly all months. And depending on this result, the forecasted values (using model in Table 1) can be observed. Fig. 3 represents the results of 2 years forecasting.

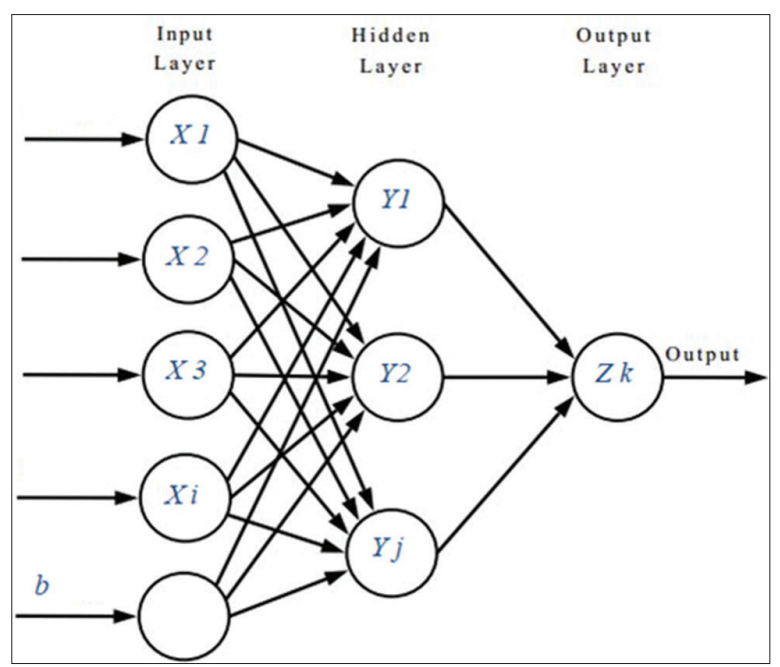

Fig. 3. Artificial neural network structure used in midterm load forecasting.

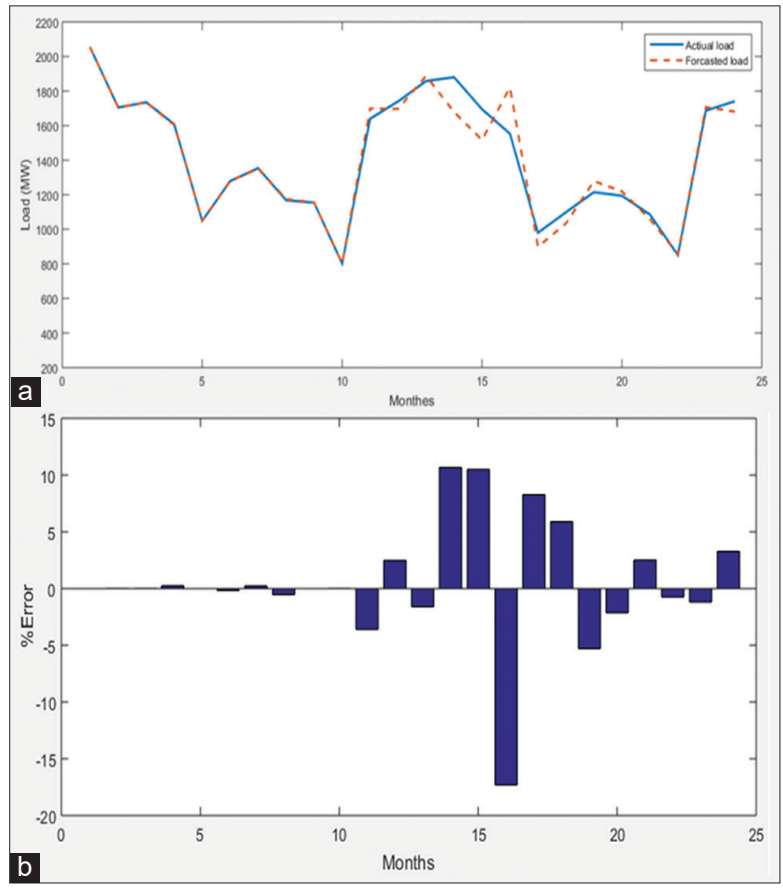

Fig. 4. (a) Actual and forecasted load demand for 24 months (b) percentage error for 24 months. 
Fig. 4(a) and (b) shows the value of actual load demand in 2016-2017 on dash line. Solid line shows the forecasted values. The mean absolute percentage error is $5.6 \%$.

Using the model developed, load, error, and \% error of all the months have been calculated. Then, error and $\%$ error are found out using the below given formula.

$$
\begin{aligned}
& \text { Error }=\text { Output by NN }- \text { Actual output } \\
& \% \text { Error }=(\text { Error } / \text { actual output }) \times 100
\end{aligned}
$$

Actual output, output by NN, error, and \% Error in 2016 and 2017 are given in Tables 2 and 3.

$\begin{aligned} & \text { TABLE 2: Percentage error of different cases for } \\
& \text { January } \mathbf{2 0 1 3}\end{aligned}$
\begin{tabular}{lclc} 
No. of hidden layer & Neurons & Transfer function & PE \\
\hline 1 & 10 & LOGSIG & -53.5031 \\
12 & & -25.4602 \\
& 15 & & -25.4602 \\
1 & 10 & TANSIG & 0 \\
& 12 & & -119.092 \\
& 15 & & 0 \\
2 & 10 & LOGSIG & -0.33203 \\
& 12 & & -40.0727 \\
& 15 & & -56.5244 \\
\hline
\end{tabular}

\section{TABLE 3: Actual, forecasted load demand, and} percentage error

\begin{tabular}{lccc}
\hline Month & Actual demand & Forecasted demand & \%error \\
\hline 1 & 2050 & 2050 & 0 \\
2 & 2176 & 2175.797 & 0.011883 \\
3 & 2179 & 2178.866 & 0.00774 \\
4 & 1839 & 1835.985 & 0.249687 \\
5 & 1199 & 1199 & 0 \\
6 & 1393 & 1395.096 & -0.16384 \\
7 & 1524 & 1520.822 & 0.234717 \\
8 & 1468 & 1474.335 & -0.54235 \\
9 & 1324 & 1324 & 0 \\
10 & 1421 & 1420.9246 & 0.009407 \\
11 & 1640 & 1699 & -3.59756 \\
12 & 1740 & 1696.65 & 2.491406 \\
13 & 2119 & 2149.73 & -1.60011 \\
14 & 2119 & 1918.241 & 10.67865 \\
15 & 2086 & 1907.967 & 10.5034 \\
16 & 1908 & 2179.83 & -17.3104 \\
17 & 1440 & 1358.92 & 8.273469 \\
18 & 1102 & 1037 & 5.914468 \\
19 & 1268 & 1332.29 & -5.29136 \\
20 & 1194 & 1219.61 & -2.14489 \\
21 & 1390 & 1362.76 & 2.508287 \\
22 & 1391 & 1397.36 & -0.74736 \\
23 & 1191 & 1211 & -1.18554 \\
24 & 1285 & 1228.05 & 3.274871 \\
\hline & & & \\
& 1285 & & \\
\hline
\end{tabular}

(The error is different because of the different in actual output and the output in the NN).

\section{CONCLUSIONS}

ANN models provide a very useful tool for midterm load forecasting. Radically different from statistical methods, these models have shown promising results in load forecasting. The aim of this paper is to develop a practical model for the peak load demand of Sulaimani Governorate which gives a best expectation values with minimum errors so that directorate of central control planners can estimate what the near-term demand will be. The monthly peak demand estimation is a key input in determining if there is enough generating capacity available to meet the demand.

The result of this research shows the high efficiency of the neural network in estimating the electrical power load and this is because ANN can define the nonlinear relation between the weather data and the load with high accuracy.

Midterm monthly load forecasting using ANN can lead to very good results if the ANN structure is well designed and training data selection is appropriate.

As it can be seen on the table above, the electricity demand is increasing to the peak in January, February, and December every year. From this point, Sulaimani city needs extra investment on electricity energy load to satisfy the demand of consumption. In 2015 and 2016 years, electricity demand is above 2000 MW in January.

In this work, Sulaimani maximum electrical energy demand has been forecasted by considering different ANN models with high accuracy, to model the effects of weather, proper ANN models have been implemented. Because any prediction model does not give the best results, nine different ANN models for prediction were performed with the same period data and the superior ANN model was detected for forecasting electricity demand.

Forecasts model can be varied number of neuron in hidden layer 10,12, and 15 neurons. The results show 2 years ahead midterm load forecasting model of 12 neurons in hidden layer can be reduced error. MAPE in this model is 5.6\%.

This research and generally every research about load forecasting can be helpful for scheduling on requirement on 
developing electrical distribution network, switching, selling energy, maintenance, and repairmen.

To have even better results, we may need to have more sophisticated topology for the neural network which can discriminate start-up months from other months. Here, we utilized only temperature, humidity, and precipitation among other weather information. Nevertheless, ANN may enable us to model such weather information for midterm procedure. The use of additional weather variables such as cloud coverage and wind speed should yield even better result.

\section{ACKNOWLEDGMENT}

The author would like to thank the staff Directory of Sulaimani Dispatch Control Center, especially operation department, and Sulaimani Directory of Metrological and Seismology data for the contribution of this work and providing.

\section{REFERENCES}

[1] H. S. Hippert, C. E. Pedreira and R. C. Souza. "Neural Networks for Short-term Load Forecasting: A Review and Evaluation". Vol. 16. In: IEEE Transactions on Power Systems, Piscataway, New Jersey, 2001.

[2] United Nations Development Programme. "Electricity Network Development Plan Sulaimani Governorate, UNDP-ENRP, Distribution Sector Revision 1 February". United Nations Development Programme, New York. 2002.

[3] A. Mohan. "Mid term electrical load forecasting for state of Himachal Pradesh using different weather conditions via ANN model". International Journal of Research in Management, Science and Technology, vol. 1, no. 2, 80, 2013.

[4] M. R. G. Al-Shakarchi and M. M. Ghulaim. "Short-term load forecasting for baghdad electricity region. Electric Machines and
Power Systems, vol. 28, pp. 355-371, 2000.

[5] S. H. Ling, F. H. F. Leung, H. K. Lam and P. K. S. Tam. "Short-term Electric Load Forecasting Based on a Neural on a Neural Fuzzy Network". Vol. 50. In: IEEE Transactions on Industrial Electronics, 2003.

[6] G. C. Liao and T. P. Tsao. "Integrated genetic algorithm/Tabu search and neural fuzzy networks for short-term load forecasting". Power Engineering Society General Meeting, vol. 1, pp. 1082-1087, 2004.

[7] P. K. Dash, S. Mishra, S. Dash, A. C. Liew. "Genetic Optimization of a Self-organizing Fuzzy-Neural Network for Load Forecasting". In: IEEE Power Engineering Society Winter Meeting, Conference Proceedings, 2000.

[8] United States Agency for International Development. Electricity Sector Master Plan for Iraq. United States Agency for International Development, Washington, DC, United States, 2004.

[9] B. Islam. "Comparison of conventional and modern load forecasting techniques based on artificial intelligence and expert systems". IJCSI International Journal of Computer Science Issues, vol. 8, no. 3, pp. 504-513, 2011.

[10] G. B. Huang, Q. Y. Zhu, K. Mao, C. K. Siew, P. Saratchandran and N. Sundararajan. "Can threshold networks be trained directly". Vol. 53. In: IEE Transactions on Circuits and Systems Part 2: Express Briefs, pp. 187-191, 2006.

[11] A. Nahari, H. Rostami, R. Dashti. "Electrical load forecasting in power distribution network by using artificial neural network". International Journal of Electronics Communication and Computer Engineering, vol. 4, no. 6, 2013.

[12] Y. Y. Hsu and C. C. Yang. "Design of Artificial Neural Networks for Short-term Load Forecasting. Part I: Self-organizing Feature Maps for Day Type Selection”. Vol. 138. In: IEEE Proceedings-C, pp. 407-413, 1991.

[13] M. Djukanovic, B. Babic, D. J. Sobajic and Y. H. Pao. "Unsupervised/ Supervised Learning Concept for 24-Hour Load Forecasting". Vol. 140. In: IEE Proceedings-C, pp. 311-318, 1993.

[14] Y. Wang and D. Gu. "Back Propagation Neural Network for Short-term Electricity Load Forecasting with Weather Features". In: International Conference on Computational Intelligence and Natural Computing, 2009.

[15] M. Buhari and S. Adamu. "Short Term Load Forecasting Using Artificial Neural Naetwork". Vol. 1. In: Proceeding of the International Multi Conference of Engineering and Computer Scientists, pp. 221-226, 2012. 